J. Clin. Chem. Clin. Biochem.

Vol. 17, 1979, pp. 85-88

\title{
Zum Nachweis des neuen Analgetikums Tramadol $\left.\left.^{1}\right)\left(\text { Tramal }^{\circledR}\right)^{2}\right)$
}

\author{
Von H. Schütz
}

Aus dem Institut für Rechtsmedizin (Leitung: Prof. Dr. Dr. G. Schewe) der Universität Gießen

(Eingegangen am 15. Juni/6. September 1978)

Herrn Professor Dr. Dr. h. c. H. Böhme zum 70. Geburtstag in Verehrung gewidmet

Zusammenfassung: Analytische Daten (DC, GLC, UV, IR, MS) des neuen Analgetikums Tramadol werden beschrieben. Weiterhin wird über die Extraktion biologischer Proben und erste Ergebnisse der Untersuchungen zur Biotransformation berichtet.

\section{Detection of the new analgetic Tramadol (Tramal ${ }^{\circledR}$ )}

Summary: Analytical data (TLC, GLC, UV, IR, MS) of the new analgetic, Tramadol are described. Extraction of the material from biological samples and preliminary results on its biotransformation are also reported.

\section{Einleitung}

Nach Nefopam (Ajan ${ }^{\circledR}$ ), über dessen Nachweis und Biotransformation wir vor einiger Zeit berichteten $(1,2)$, wurde kürzlich $(3,12-29)$ erneut ein starkes Analgetikum eingeführt, das wiederum von beträchtlichem chemisch-toxikologischem Interesse ist (Untersuchungen im Rahmen von Abhängigkeits- und Vergiftungsfällen, verkehrsmedizinische Bedeutung im Hinblick auf die vom Hersteller angegebene Beeinflussungsmöglichkeit des Reaktionsvermögens, insbesondere in Verbindung mit Alkohol (4)).

Es handelt sich hierbei um das Aminomethylcyclohexanolderivat Tramadol $\left.\left(\text { Tramal }^{\circledR}\right)^{3}\right)$ mit der nachfolgend wiedergegebenen Struktur (Abb. 1):<smiles>COc1cccc(C2(O)CCCCC2CN(C)C)c1</smiles>

Abb. 1. Formel von Tramadol (Tramal ${ }^{\circledR}$ ).

Über die chemischen und physikochemischen Eigenschaften $(13,14)$, die Pharmakologie $(13,15-18)$, die Toxikologie (19) und die klinische Wirkung (20-29) von

\footnotetext{
1) = (t)-trans-2-(dimethylamino-methyl):1=(m-methoxy-phenyl $)-$ cyclohexanol.

2) = ( \pm )-trans-2-(dimethylamino-methyl $)-1-(m-$ methoxy-phenyl $)-$ cyclohexanol-hydrochlorid.

3) Wir danken Herrn Prof. Dr. Flohé (Grünenthal GmbH) für die freundliche Überlassung von Substanzmustern.
}

Tramadol wurde ausführlich berichtet, während zur Biotransformation und Pharmakokinetik bislang nur qualitative Angaben veröffentlicht wurden (30). Da sich in der chemisch-toxikologischen Praxis das Problem des Nachweises jedoch bereits kurze Zeit nach der Neueinführung eines Präparates stellt, leiteten wir Untersuchungen zur chemisch-toxikologischen Analytik ein, über deren Ergebnisse nachstehend berichtet werden soll.

$\begin{array}{ll}\text { Allgemeine Daten für Tramadol } \\ \text { Summenformel: } & \begin{array}{l}\mathrm{C}_{16} \mathrm{H}_{25} \mathrm{NO}_{2} \\ \mathrm{C}_{16} \mathrm{H}_{25} \mathrm{NO}_{2} \cdot \mathrm{HCl}\end{array} \\ \text { Molekulargewicht: } & \begin{array}{l}\text { Base: } 263,4 \\ \text { Hydrochlorid: } 299,84\end{array} \\ \text { Löslichkeit: } & \begin{array}{l}\text { Base: In den meisten organischen Lösungs- } \\ \text { mitteln gut bis sehr gut löslich }\end{array} \\ & \begin{array}{l}\text { Hydrochlorid: löslich in } 30 \text { Teilen Wasser, } \\ 35 \text { Teilen 0,1 mol/1 HCl und } 30 \text { Teilen }\end{array} \\ \text { Schmelzpunkt: } & \begin{array}{l}\text { Methanol. } \\ \text { Base: Kein definierter Fp. }\end{array} \\ \text { Handelsform: } & \begin{array}{l}\text { Ampullen: } 1 \text { ml Injektionslösung enthält } \\ 50 \text { mg Tramadol-Hydrochlorid }\end{array} \\ & \begin{array}{l}\text { Zäpfchen: } 100 \text { mg Tramadol-Hydrochlorid } \\ \text { Nur in Ausnahmefällen über } 400 \text { mg Trama- } \\ \text { dol-Hydrochlorid (4). }\end{array}\end{array}$

\section{Material und Methoden}

Dünnschichtchromatographische Untersuchungen Trägermaterial

DC-Fertigplatten Kieselgel $60 \mathrm{~F}_{254}$ (E. Merck, Darmstadt), aufsteigende Methode, Kammersättigung, keine besondere Aktivierung der DC-Platten. 
Tab. 1. Dünnschichtchromatographie von Tramadol und anderen gebräuchlichen Pharmaka.

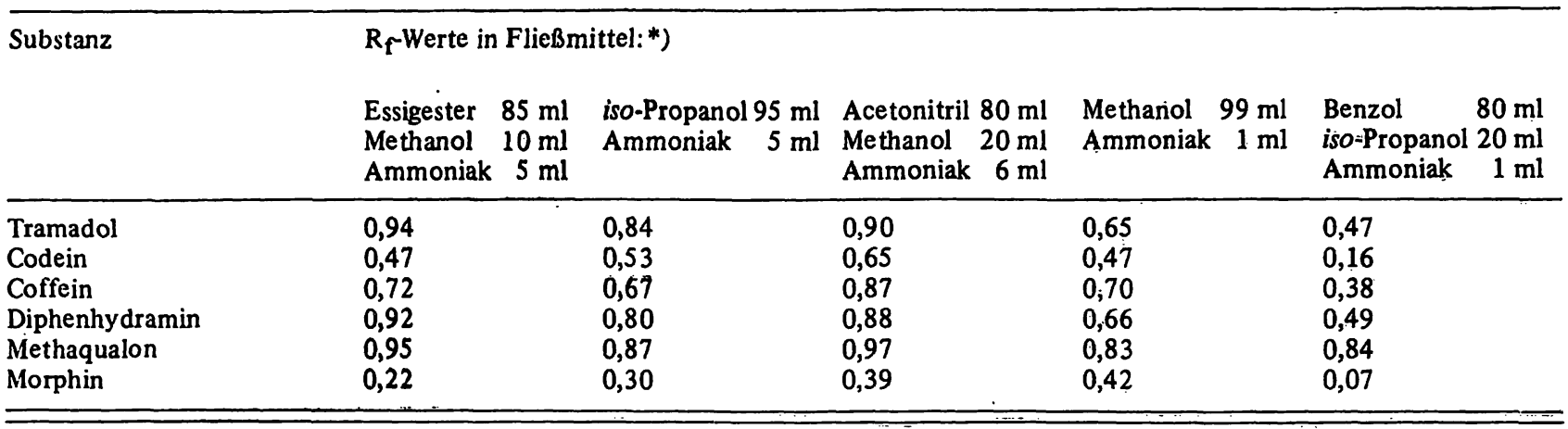

Dünnschichtchromatographie von Tramadol und zwei Hauptmetaboliten (Desalkylierungsprodukte)

\begin{tabular}{lll} 
Substanz & $\begin{array}{l}\text { Benzol } 80 \mathrm{ml} \\
\text { iso-Propanol } 20 \mathrm{ml}\end{array}$ & $\begin{array}{l}\text { Eine Detektion der Metaboliten ist ebenfalls mit Dragendorff-Reagenz (gelbbraun) } \\
\text { bzwaliumiodoplatinat (violett) möglich. }\end{array}$ \\
Ammoniak $3 \mathrm{ml}$ & \\
Tramadol & 0,88 & \\
Metabolit I & 0,67 & \\
Metabolit II & 0,31 & \\
\hline
\end{tabular}

*) Bei der Angabe der Fließmittelzusammensetzung bedeutet Ammoniak: Ammoniaklösung ( $250 \mathrm{~g} / \mathrm{kg}$ )

\section{Fließmittel}

Vgl. Tabelle 1.

Als Referenzsubstanzen wurden die häufig zu diesem $Z$ weck benutzten Wirkstoffe Codein, Coffein, Diphenhydramin und Methaqualon benutzt. Weiterhin wurde Morphin als starkes Analgetikum vergleichsweise mitgeführt.

Detektionsmöglichkeiten

Trocknen der entwickelten DC-Platte bei $50^{\circ} \mathrm{C}$ während etwa $10 \mathrm{~min}$.

1. Dragendorff-Reagenz nach Munier und Macheboeuf (vgl. Stahl (5), S. 829, Nr. 89): Gelbbraune Farbtönung mit Tramadol.

2. Kaliumiodoplatinat (vgl. Stahl (5), S. 837, Nr. 140):

Violette Anfärbung mit Tramadol.

3. Folin-Ciaucalteaus-Reagenz (vgl. Stahl (5), S. 832,

Nr. 108): Blauviolette Farbtönungen insbesondere mit phenolischen Metaboliten.

Die Trennergebnisse sind in Tabelle 1 wiedergegeben.

Gaschromatographische Untersuchungen

Gerät: Varian Aerograph Series $2100 \mathrm{mit}$ FID; OT $190^{\circ} \mathrm{C}$,

ET $220^{\circ} \mathrm{C}$, FID-Zuleitung $250^{\circ} \mathrm{C}$; Säule: Etwa $1,5 \mathrm{~m}$ Glas,

$2 \mathrm{~mm}$ ID mit $3 \%$ OV-17 bzw. 3\% SE-30 jeweils auf Chromosorb

G, AW/DMCS (80/100 mesh); Trägergas: Stickstoff

$\left(\mathrm{P}_{2}=\right.$ etwa $\left.98 \mathrm{kPa}\right)$.

UV-Photometrie

Gerät: Unicam S. P. 800 (Kalibrierung mit Holmium, Quarzküvetten mit $\mathrm{d}=1 \mathrm{~cm}$, p. a. Reinheitsgrade der Lösungsmittel).

Substanzkonzentrationen: Etwa $20 \mathrm{mg} / \mathrm{l}$.

IR-Spektroskopie

Gerät: Perkin Elmer Typ 337, Standard-Preßling für Base und Hydrochlorid, etwa $1 \mathrm{mg}$ Substanz pro $300 \mathrm{mg} \mathrm{KBr}$ (UVASOL). Mittlere Registriergeschwindigkeit, Slit $=\mathrm{N}$.

Massenspek trum $\cdots$

Gerät: Värian MAT 101 (Direkteinlaß, Ionenquelle $66^{\circ} \mathrm{C}$, Ionisierungsenergie $70 \mathrm{eV}$ ).

Extraktion des Harnes

Jeweils $100 \mathrm{ml}$ eines 12-Stunden Sammelharnes wurden bei pH 11 mit Chloroform (Verhältnis Urin zu Extraktionsmittel $1+1)$ ausgeschüttelt.

Zur Konjugatspalting wurde bei pH 5,5 und $37^{\circ} \mathrm{C}$ etwa 18 Stunden mit Arylsulfatase/ $\beta$-Glucuronidase ( $5 \mathrm{ml} / 1$ Urin) inkubiert. Anschließend erfolgten Extraktionen bei pH 9 mit Chloroform/iso-Propanol (Volumina $40 \mathrm{ml}+10 \mathrm{ml}$ ). Die Extrakte wurden im Vakuumrotationsverdampfer bei $30^{\circ} \mathrm{C}$ schonend eingeengt und bis zur Analyse tiefgefroren aufbewahrt.

\section{Ergebnisse und Diskussion}

\section{Dünnschich tchromatographische Untersuchungen}

Die Trennergebnisse sind in Tabelle 1 wiedergegeben. Die Detektion erfolgte mit den beschriebenen Detektionsmitteln (zur Indizierung von Coffein wurde die Fluoreszenzminderung bei $254 \mathrm{~nm}$ herangezogen).

\section{Gaschromatographische Untersuchungen}

Trennergebnisse

$\begin{array}{lll}\text { Substanz } & \begin{array}{l}\text { Netto- } \\ \text { retentionszeit }\end{array} & \begin{array}{l}\text { Retentions- } \\ \text { index }\end{array}\end{array}$

OV-17

Tramadol $227 \mathrm{~s} \quad 2230$

Diphenhydramin $\quad 151 \mathrm{~s} \quad 2155$

SE-30

Tramadol $\quad 160 \mathrm{~s} \quad 1945$

Diphenhydramin $\quad 123 \mathrm{~s} \quad 1880$

\section{UV-Photometrie}

Die UV-Spektren sind in Abbildung 2 wiedergegeben. Daten:

In Ethanol abs.:

Maxima bei $273 \mathrm{~nm}$ und $279 \mathrm{~nm}$, Minimum bei $242 \mathrm{~nm}$

in $0,1 \mathrm{~mol} / 1 \mathrm{HCl}$ :

Maxima bei $272 \mathrm{~nm}$ und $277 \mathrm{~nm}$, Minimum bei $242 \mathrm{~nm}$

$0,1 \mathrm{~mol} / 1 \mathrm{NaOH}$ :

Maxima bei $272 \mathrm{~nm}$ und $277 \mathrm{~nm}$, Minimum bei $240 \mathrm{~nm}$.

\section{IR-Spektren}

Die IR-Spektren sind in Abbildung 3 (Tramadol-Hydrochlorid) bzw. Abbildung 4 (Tramadol-Base) wiedergegeben. 


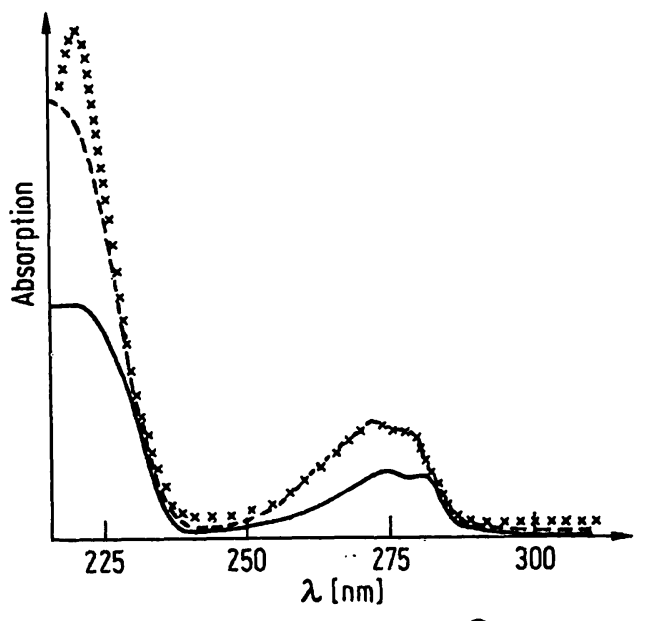

Abb. 2. UV-Spektren von Tramadol (Tramal $\left.{ }^{\circledR}\right)$.

$-=$ in Ethanol abs.,

$----=$ in $0,1 \mathrm{~mol} / 1 \mathrm{HCl}$,

$x \times \times x=$ in $0,1 \mathrm{~mol} / 1 \mathrm{NaOH}$.

UNICAM S. P. 800 (Kalibrierung mit Holmium).
Massenspektrum ${ }^{4}$ )

Das Massenspektrum von Tramadol ist in Abbildung 5 wiedergegeben.

\section{Interpretationsmöglichkeiten}

Beachtenswert ist der stark ausgeprägte Peak für das Molekülion. Für die Interpretation der übrigen Peaks bieten sich (vorbehaltlich einer Absicherung durch Hochauflösungswerte) folgende Möglichkeiten an: $\mathrm{m} / \mathrm{e}=218$ : Verlust von Dimethylamin. $\mathrm{m} / \mathrm{e}=200$ : $\mathrm{H}_{2} \mathrm{O}$-Abspaltung aus $\mathrm{m} / \mathrm{e}=218 . \mathrm{m} / \mathrm{e}=188$ : Verlust von Formaldehyd. $\mathrm{m} / \mathrm{e}=173$ : anschließende Abspaltung von Methyl, bzw. $\mathrm{m} / \mathrm{e}=160$ : anschließende Abspaltung von Ethylen. $\mathrm{m} / \mathrm{e}=135: m$-Methoxybenzoylkation (häufig bei Benzylalkoholen). $\mathrm{m} / \mathrm{e}=84$ :

$\mathrm{CH}_{2}=\mathrm{CH}-\mathrm{CHN}_{\stackrel{+}{-}}-\mathrm{CH}_{\mathrm{CH}_{3}} \cdot \mathrm{m} / \mathrm{e}=77: \mathrm{C}_{6} \mathrm{H}_{5}^{+} \cdot \mathrm{m} / \mathrm{e}=107$ :

Methoxyphenyl.

4) Mein besonderer Dank gilt Herrn Prof. Dr. G. Spiteller für seine Unterstützung bei der Abfassung dieses Abschnittes.

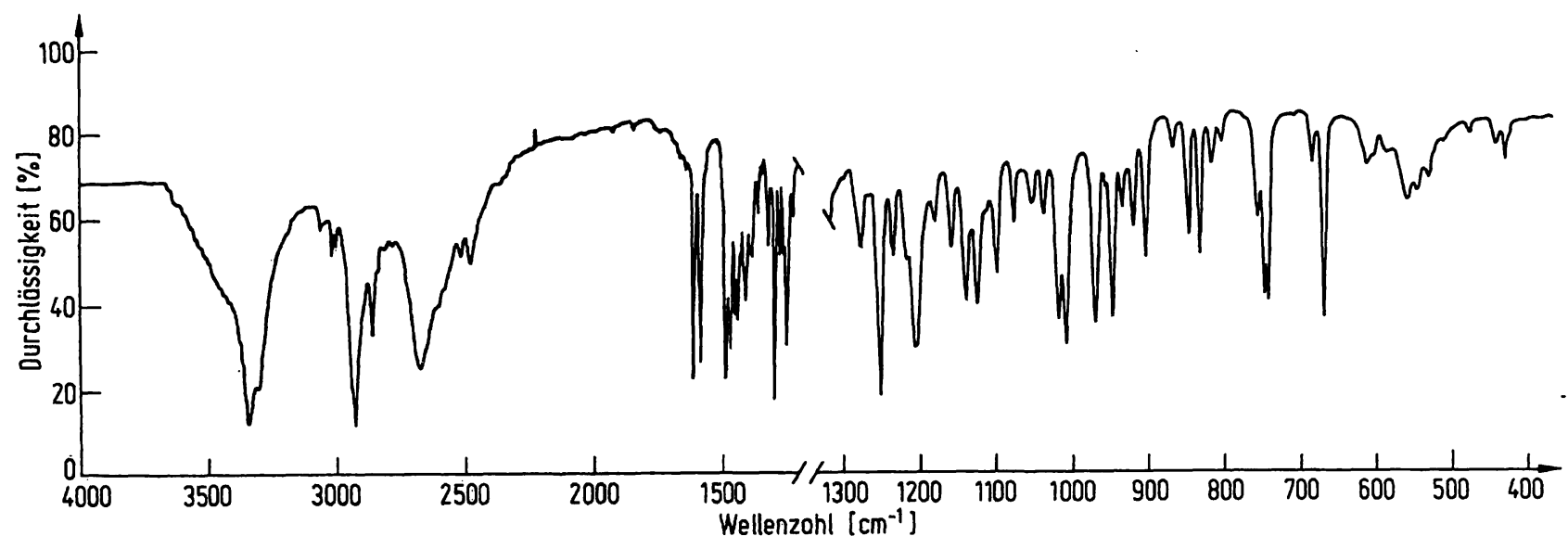

Abb. 3. IR-Spektrum von Tramadol-Hydrochlorid PERKINELMER 337 (KBr-Technik). Einige wichtige Bandenzuordnungen lauten:
$3340 \mathrm{~cm}^{-1}: \quad \nu-\mathrm{OH}$
ca. $2900 \mathrm{~cm}^{-1}: \nu_{\text {as. }}-\mathrm{CH}_{2}-$ bzw. $-\mathrm{CH}_{3}$

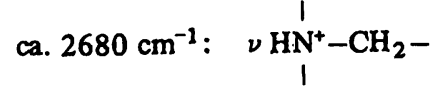
ca. $1600 \mathrm{~cm}^{-1}: \quad \nu-\stackrel{C}{\mathrm{C}=\mathrm{C}}-$ (arom.)

$$
\begin{aligned}
& \text { ca. } 1240 \mathrm{~cm}^{-1}: \nu_{\text {as. }}-\stackrel{\text { I }}{\mathrm{C}}-\mathrm{O}-\mathrm{CH}_{3} \\
& \text { ca. } 1040 \mathrm{~cm}^{-1}: \quad \nu-\mathrm{C}-\mathrm{O}-\mathrm{CH}_{3} \\
& \text { ca. } 700-800 \mathrm{~cm}^{-1} \delta-\stackrel{l}{\mathrm{C}}-\mathrm{H} \text { (Benzolring) }
\end{aligned}
$$

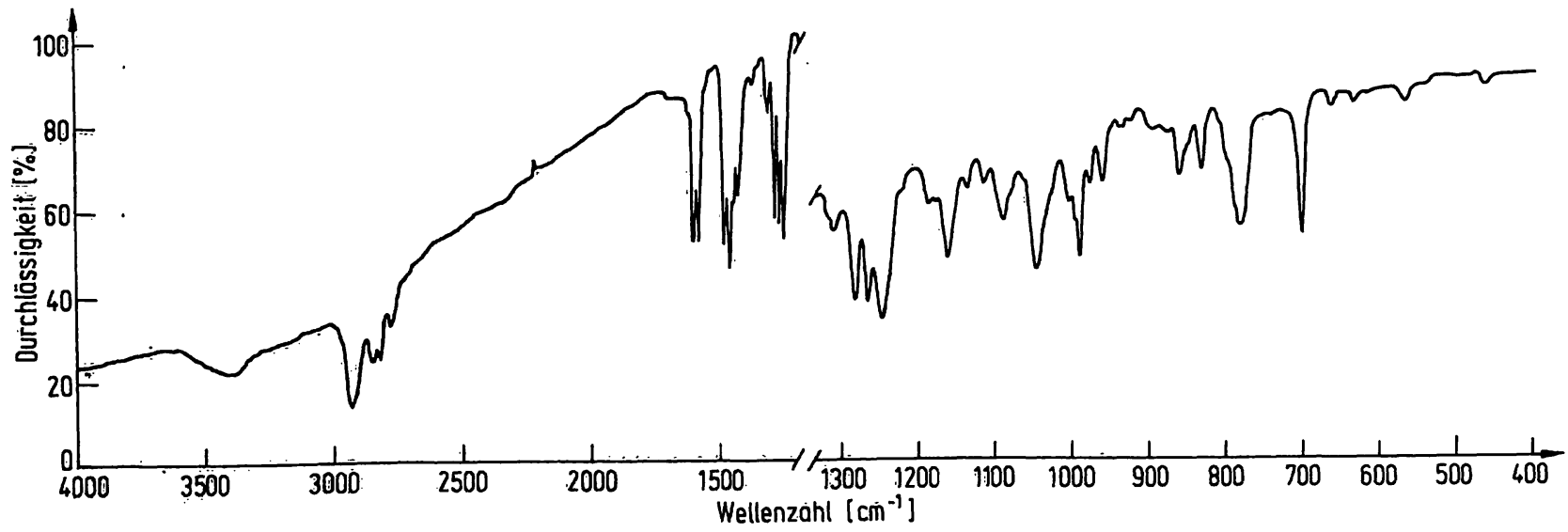

Abb. 4. IR-Spektrum der Tramadol-Base PERKIN ELMER 337 (KBr-Technik). 


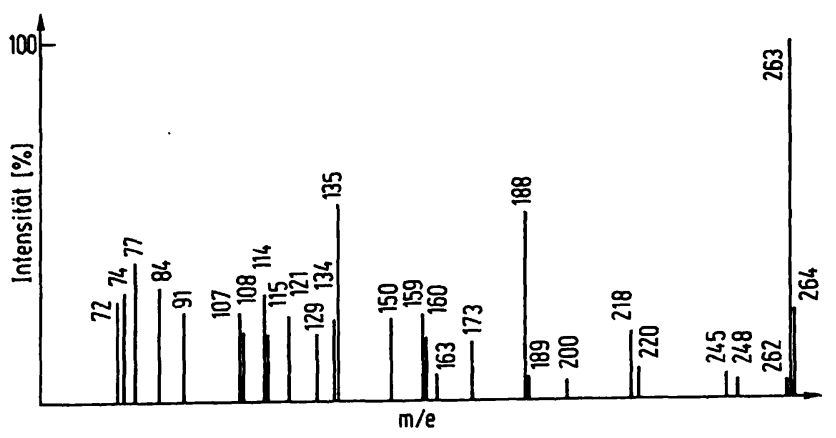

Abb. 5. Massenspektrum von Tramadol (Tramal ${ }^{\circledR}$ ). VARIAN MAT 101 (Direkteinlaß, Ionenquelle $66^{\circ} \mathrm{C}, 70 \mathrm{eV}$ ).

Ergebnisse der Untersuchungen zur Biotransformation und Ausscheidung im Harn

In der vorliegenden Arbeit soll das Hauptgewicht auf der analytischen Seite liegen, um Daten zum Nachweis von Tramadol zur Verfügung stellen zu können. Die ausführliche Darstellung der noch nicht abgeschlossenen Untersuchungen zur Biotransformation und Pharmakokinetik bleibt einer späteren Publikation vorbehalten. Dennoch sollen nachstehend einige vorläufige Ergebnisse zu diesen Fragestellungen mitgeteilt werden:

1. Nach einer einmaligen Applikation von $100 \mathrm{mg}$ Tramadol-Hydrochlorid $\left(=\mathrm{Tramal}^{\mathrm{R}}\right)$ als Zäpfchen konnte in $100 \mathrm{ml}$ des ersten 12-Stunden-Sammelurins unverändertes Tramadol in einer Größenordnung von $500-1000 \mu \mathrm{g} / 100 \mathrm{ml}$ gaschromatographisch nachge-

\section{Literatur}

1. Ebel, S. \& Schütz, H. (1977), Arch. Toxicol. 38, 239-250.

2. Ebel, S. \& Schütz, H. (1978), Arch. Pharm. (Weinheim Ger.) $311,547-551$.

3. Pharmazeutische Stoffliste, 4. Aufl., 61. Erg. Lfg., S. 1599, bearb. u. hrsg. vom Arzneimittelbüro der ABDA, Werbeund Vertriebsgesellschaft Deutscher Apotheker mbH, Frankfurt/M.

4. Beipackzettel zu Tramadol $\left(\operatorname{Tramal}^{\circledR}\right)$, Fa. Grünenthal GmbH, Stolberg.

5. Stahl, E. (1967), Dünnschichtchromatographie, 2. Aufl., Springer-Verlag, Berlin-Heidelberg-New York.

6. Geldmacher-von Mallinckrodt, M. (1976), Einfache Untersuchungen auf Gifte im klinisch-chemischen Laboratorium, Thieme, Stuttgart, S. 157.

7. Schütz, H. W. (1971), Beitr. Gerichtl. Med. 28, 354-358.

8. Kováts, E. (1958), Helv. Chim. Acta 41, 1915.

9. Kaiser, R. E. (1974), Chromatographia 7, 251-257.

10. Post, D. (1976), Beitr. Gerichtl. Med. 34, 219-227.

11. Dr. Lintz, Grünenthal $\mathrm{GmbH}$, Stolberg (pers. Mitteilung).

12. Flohé, L. \& Friderichs, E. (1978), Arzneim. Forsch. (Drug Res.) 28, 99-106.

13. Flick, K., Frankus, E. \& Friderichs, E. (1978), Arzneim. Forsch. (Drug Res.) 28, 107-113.

14. Frankus, E., Friderichs, E., Kim, S. M. \& Osterloh, G, . (1978), Arzneim. Forsch. (Drug Res.) 28, 114-121.

15. Friderichs, E., Felgenhauer, F., Jongschaap, P. \& Osterloh G. (1978), Arzneim. Forsch. (Drug Res.) 28, 122-134.

16. Osterloh, G., Friderichs, E., Felgenhauer, F., Günzler, W. A., Henmi, Z., Kitano, K., Nakamura, M., Hayashi, H. \& Ishii, I. (1978), Arzneim. Forsch. (Drug Res.) 28, $135-151$. wiesen werden. $\mathrm{Zu}$ diesem $\mathrm{Z}$ weck wurde der bei $\mathrm{pH} 11$ mit Chloroform gewonnene Basenextrakt (vgl. Methodik) untersucht. Die beschriebene Methodik ist hinreichend empfindlich, um noch 100 Stunden nach der Applikation unveränderten Wirkstoff (Größenordnung 500$1000 \mathrm{ng} / 100 \mathrm{ml}$ ) zu identifizieren.

Diese Befunde entsprechen den vorläufigen Angaben des Herstellers (30) zur Ausscheidungskinetik von Tramadol.

2. Die Untersuchung des Basenextraktes lieferte weiterhin Hinweise auf die renale Ausscheidung von zwei Metaboliten (vgl. Tab. 1). Nach ersten spektroskopischen Ergebnissen handelt es sich dabei um ein Mono- bzw. Didesalkylierungsprodukt von Tramadol.

Die Richtigkeit dieser Vermutung wurde uns zwischenzeitlich bestätigt (11). Nach Auskunft des Herstellers konnten die Metabolite O-Desmethyl-, N-Desmethylund $\mathrm{O}, \mathrm{N}$-Bisdesmethyl-Tramadol náchgewiesen werden $(11,30)$.

3. Im Phenolbasenextrakt nach der enzymatischen Hydrolyse traten ferner zahlreiche phenolische Metaboliten auf, die sich mit Folin-Ciaucalteaus-Reagenz (aber auch mit Dragendorff-Reagenz und Kaliumiodoplatinat) anfärben ließen. Diese Substanzen konnten erst nach der Konjugatspaltung extrahiert werden, lagen vorher demnach offensichtlich als Glucuronide oder Sulfate vor. Eine Strukturaufklärung ist eingeleitet, aber noch nicht abgeschlossen.

17. Murano, T., Yamamoto, T., Endo, N., Kudo, Y., Okada, N., Masuda, Y. \& Yano, I. (1978), Arzneim. Forsch. (Drug Res.) 28, 152-158.

18. Yanagita, T. (1978), Arzneim. Forsch. (Drug Res.) 28, 158-163.

19. Lagler, F., Helm, F., Etzel, V. \& Kiel, H. (1978), Arzneim. Forsch. (Drug Res.) 28, 164-172.

20. Krueger, H. \& Fazel-Madjlessi, A. (1978), Arzneim. Forsch. (Drug Res.) 28, 173-176.

21. Krueger, H. \& Müller-Limmroth, W. (1978), Arzneim. Forsch. (Drug Res.) 28, 176-178.

22. Müller-Limmroth, W. \& Krueger, H. (1978), Arżneim. Forsch. (Drug Res.) 28, 179-180.

23. Rost, A. \& Schenck, E. G. (1978), Arzneim. Forsch. (Drug Res.) 28, 181-183.

24. Vogel, W., Burchardi, H., Sihler, K. \& Valiĉ, L. (1978), Arzneim. Forsch. (Drug Res.) 28, 183-186.

25. Friedel, B. (1978), Arzneim. Forsch. (Drug Res.) 28, $187-189$.

26. Huber, H. P. (1978), Arzneim. Forsch. (Drug Res.) 28, 189-191.

27. Arend, I., von Arnim, B., Nijssen, J. \& Scheele, J. (1978), Arzneim. Forsch. (Drug Res.) 28, 199-208.

28. Schenck, E. G. \& Arend, I. (1978), Arzneim. Forsch. (Drug Res.) 28, 209-212.

29. Flohé, L., Arend, I., Cogal, A., Richter, W. \& Simon, W. (1978), Arzneim. Forsch. (Drug Res.) 28, 213-217.

30. Informationsschrift der Grünenthal GmbH: Tramal ${ }^{\mathrm{R}}$; stark wirksames Analgetikum, Stolberg, 1978.

Dr. rer. nat. Harald Schütz Institut für Rech tsmedizin der Justus Liebig-Universität Frankfurter Straße 58. D-6300 Gießen 